\title{
Type 1 diabetes mellitus following anti-programmed cell death-1 therapy
}

\section{K. Clotman ${ }^{1}$, K. Janssens ${ }^{2}$, P. Specenier ${ }^{2,3}$, I. Weets ${ }^{4}$, C. De Block ${ }^{1,2}$}

${ }^{1}$ Department of Endocrinology, Diabetology \& Metabolism, Antwerp University Hospital, Belgium

2 University of Antwerp, Belgium

${ }^{3}$ Department of Oncology, University Hospital Antwerp, Belgium

${ }^{4}$ Diabetes Research Center, Brussels Free University (VUB), Belgium
K. Clotman and K. Janssens contributed equally to this work

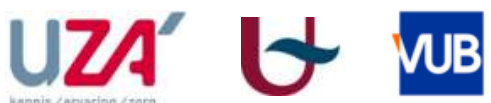

\section{Background}

Cancer immunotherapy is a successful and fast growing field. Pembrolizumab (Keytruda) and nivolumab (Opdivo) are approved for malignant melanoma and several other cancer types. They are two IgG4 monoclonal antibodies targeting the programmed cell death-1 (PD-1) receptor. This receptor is important in maintaining selftolerance and is therapeutically targeted by immune checkpoint inhibiting monoclonal antibodies (mAb) to enhance antitumor immune responses. However, immune checkpoint blockade is associated with a risk for immune-related adverse events (irAE) potentially affecting the endocrine organs. Auto-immune diabetes mellitus and the associated diabetic ketoacidosis (DKA) are examples of rare irAEs.

Since the use of immunotherapy is expected to increase, the incidence of immune-related adverse events is also expected to rise. Therefore, it is essential that all clinicians are aware of diabetic ketoacidosis as a rare and life-threatening side effect of immunotherapy.

\section{Aims}

To provide a timely review of all listed cases of antiPD-1 induced autoimmune diabetes, discuss pathogenetic mechanisms, and propose a screening and management protocol.

\section{Methods}

A literature search was conducted on Pubmed and Web of Science with the following keywords:

- diabetes mellitus

- cancer immunotherapy

- anti-PD-1 therapy

- pembrolizumab

- nivolumab

\section{Results}

So far 38 cases of anti-PD1-induced type 1 diabetes mellitus have been reported. The incidence of autoimmune diabetes in patients receiving pembrolizumab monotherapy is $0.2 \%$, and $0.9 \%$ in nivolumab monotherapy. However, the incidence increases to $1.5 \%$ in nivolumab and ipilimumab combination therapy.

Patients presented with variable symptoms, ranging from asymptomatic hyperglycemia, to polyuria and polydipsia to severe diabetic ketoacidosis. Diabetic ketoacidosis was the first sign of diabetes in 25 out of 34 (73.5\%) reported cases. Time from initiation of anti-PD1 therapy to diagnosis of diabetes mellitus ranged from 1 week to 52 weeks and this corresponded to 1 to 17 infusions of immune checkpoint inhibitors. The median age at diagnosis was 61 years (range 28-83), an atypical age of onset for type 1 diabetes mellitus. C-peptide was low or undetectable in 28 of 30 tested patients. HbA1c levels varied from $6.4 \%$ to $10.7 \%$ ( $46-93 \mathrm{mmol} / \mathrm{mol})$. The low or undetectable c-peptide combined with the moderately low $\mathrm{HbA1c}$ levels probably indicate the fulminant onset of diabetes with rapid $\beta$-cell destruction and a shorter duration of hyperglycemia. Every patient had to be treated with intensive insulin therapy.

Eleven out of 16 tested patients had a high risk HLA type (HLA DR3-DQ2, HLA DR4-DQ8). We hypothesize that patients with high risk HLA types have an increased risk for the development of immune checkpoint inhibitor induced diabetes.

There is no clear pattern of diabetes related autoantibodies. Approximately half of the tested cases (19/34 or $55.9 \%$ ) had detectable diabetes related autoantibodies. In those 19 cases, glutamic acid decarboxylase antibodies (GADA) were positive in all subjects, tyrosine phosphatase autoantibodies (IA2A) in $5 / 19$, insulin autoantibodies in 2, ICA in 2 patients, and ZnT8 antibodies in only one subject. Four other cases have been reported, however, without antibody status. The interval between the start of anti-PD-1 antibodies to the onset of autoimmune diabetes might be related to the presence or absence of GADA. The median interval from immunotherapy initiation to diagnosis of diabetes was 4 weeks in GADA positive cases versus 10 weeks in GADA negative cases (data from 32 patients).
Table 1. Characteristics of 37 reported patients with immunotherapy associated T1DM.

\begin{tabular}{|c|c|}
\hline \multicolumn{2}{|l|}{ Causative agent } \\
\hline Nivolumab & 19 \\
\hline Pembrolizumab & 12 \\
\hline Nivolumab + ipilimumab & 2 \\
\hline Anti-PDL1 & 2 \\
\hline Anti-PD1 & 1 \\
\hline Pembrolizumab + ipilimumab & 1 \\
\hline \multicolumn{2}{|l|}{ Demographic data } \\
\hline Sex (men/women/not reported) & $18 / 18 / 1$ \\
\hline Age (years) & $61(28-83)$ \\
\hline \multicolumn{2}{|l|}{ Presentation } \\
\hline Diabetic ketoacidosis & $25(68 \%)$ \\
\hline Hyperglycemia (negative for DKA) & 3 \\
\hline HbA1c (\%) & $7.6(6.4-10.7)$ \\
\hline $\mathrm{HbA1c}(\mathrm{mmol} / \mathrm{mol})$ & $60(46-93)$ \\
\hline \multicolumn{2}{|c|}{ Time of diagnosis after start of immunotherapy } \\
\hline Number of doses & $3(1-17)$ \\
\hline Onset in weeks & $6(1-52)$ \\
\hline \multicolumn{2}{|l|}{ Beta cell antibodies } \\
\hline GADA & $19 / 34(56 \%)$ \\
\hline IA2A & $4 / 19(21 \%)$ \\
\hline ICA & $2 / 12(17 \%)$ \\
\hline IAA & $1 / 13(8 \%)$ \\
\hline ZnT8A & $1 / 6(17 \%)$ \\
\hline Undectable or low serum C-peptide & $26 / 28(93 \%)$ \\
\hline High risk HLA haplotypes & $12 / 18(67 \%)$ \\
\hline Personal history of auto-immune disease & $11 / 37(30 \%)$ \\
\hline
\end{tabular}

PD-1 reduction might cause activation of autoreactive $T$ cells resulting in autoimmune responses against pancreatic islet cells. We hypothesize that the presence of diabetes autoantibodies prior to the start of anti-PD1 therapy and certain high risk HLA types, can predispose patients to the development of immune checkpoint inhibitors related autoimmune diabetes.

We propose to educate the patients about hyperglycemic symptoms and instruct oncologists to measure glucose at each administration of a checkpoint inhibitor, thereby minimizing long delays in diagnosis and possibly avoiding the development of potentially life threatening diabetic ketoacidosis.

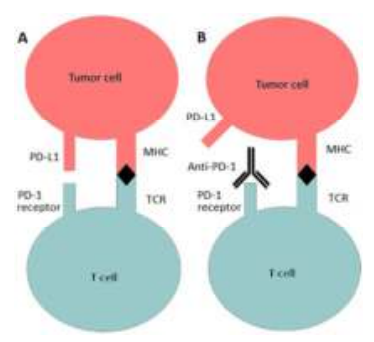

Figure 1. Mechanism of action of PD-1 immune checkpoint inhibitors

A. Tumor cells can inactivate T cells and evade the immune system by expressing PD-L1. This leads to the enhanced survival of tumor cells.

B. Anti-PD-1 can block the PD-1 receptor and restore immune response. This leads to the apoptosis of tumor cells.

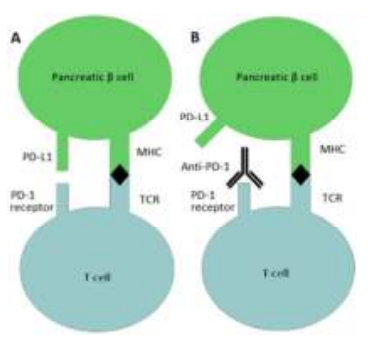

Figure 2. Hypothesis of association between PD-1 immune checkpoint inhibitors and diabetes mellitus type 1 .

A. Similar to the mechanism in figure 1 , pancreatic $\beta$ cells express PD-L1 and thereby evade immune response. // this leads to tolerance of the immune system.

B. During anti-PD-1 therapy, in certain persons, $T$ cells are activated and develop an immune response to pancreatic $\beta$ cells. // lose tolerance of pancreatic $\beta$ cells.

\section{Discussion}

Since the use of immunotherapy is expected to increase, the incidence of immune-related adverse events is also expected to rise. The incidence of autoimmune diabetes in patients receiving pembrolizumab monotherapy is $0.2 \%$, and $0.9 \%$ in nivolumab monotherapy, increasing to $1.5 \%$ in nivolumab and ipilimumab combination therapy. Therefore, it is essential that all clinicians are aware of diabetic ketoacidosis as a rare and life-threatening side effect of immunotherapy. Blood glucose monitoring during anti-PD 1 therapy is necessary. 